\title{
Association between life-course socioeconomic position and inflammatory biomarkers in older age: a nationally representative cohort study in Taiwan
}

\author{
Yu-Hsuan Lin ${ }^{1,3}$, Min-Hua Jen ${ }^{2}$ and Kuo-Liong Chien ${ }^{3^{*}}$
}

\begin{abstract}
Background: Evidence of an association between low socioeconomic position (SEP) and inflammatory markers is scant. This study aimed to examine how life-course SEP predicted C-reactive protein (CRP) and interleukin (IL-6) in older age from a national cohort.

Methods: We collected data from 1036 participants in the Social Environment and Biomarkers of Aging Study in Taiwan. Four SEP time points, childhood, young adulthood, active professional life, and older age were measured retrospectively. A group-based trajectory analysis method was used to identify the distinct trajectories of life-course SEP, and trajectory group membership was used as the predictor of CRP and IL-6 levels in older age.

Results: Three trajectories of life-course SEP were identified within the total sample: Low-Low (36.5\%), Low-High (26.8\%), and High-High (36.7\%). Participants in the High-High group had the lowest levels of CRP and IL-6.

Compared with those in the Low-Low group, the participants in the Low-High group had a similar adjusted CRP [-0.032 In mg/L; 95\% confidence interval (Cl) - 0.193, 0.128] and IL-6 (0.017 In pg/mL; $95 \% \mathrm{Cl}-0.093,0.128)$; the participants in the High-High group had a significantly lower level of adjusted CRP concentration $(-0.279 \mathrm{ln} \mathrm{mg} / \mathrm{L}$; 95\% Cl: $-0.434,-0.125)$ and similarly lower IL-6 concentration $(-0.129 \mathrm{ln} \mathrm{pg} / \mathrm{mL} ; 95 \% \mathrm{Cl}-0.236,-0.023)$.

Conclusions: Life-course SEP is related to the level of CRP and IL-6 in older age. Our data support the notion that life-course SEP predicts inflammatory markers in older age. LOW SEP in childhood is related to elevated inflammatory markers in older age. Even after the transition from low SEP in childhood to high SEP in older age, the risk remains. Further study on SEP and inflammation-related disease is warranted.
\end{abstract}

Keywords: Life-course, Socioeconomic position, Inflammation

\section{Background}

The socioeconomic position (SEP) that individuals or groups hold within the structure of society has long and widely been reported as a predictor of cardiovascular disease [1,2]. The use of inflammatory markers as predictors of cardiovascular disease has been widely studied. Markers of inflammation, in particular C-reactive protein (CRP) and interleukin 6 (IL-6),

\footnotetext{
* Correspondence: klchien@ntu.edu.tw

${ }^{3}$ Institute of Epidemiology and Preventive Medicine, College of Public Health, National Taiwan University, Mailing address: Rm. 517, 5F., No. 17, Xuzhou Rd., Zhongzheng Dist, Taipei City 100, Taiwan

Full list of author information is available at the end of the article
}

have been reported as predictors of cardiovascular diseases [3-5]. CRP is a marker of systemic inflammation [3, 6]. IL-6 acts as both a pro-inflammatory and an anti-inflammatory cytokine mediator [7]. Levels of IL-6 and CRP are physiologically linked because of the function of IL- 6 in hepatic synthesis and the excretion of CRP [7]. Evidence from observational studies suggests that low SEP is associated with inflammation, atherosclerosis, and the subsequent risk of cardiovascular disease [8-11]. Elevated levels of inflammatory markers were reported for persons of adverse life-course socioeconomic indicators. A systematic review of 25 population-based studies 
conducted in Western counties revealed inverse associations between CRP levels and SEP. The magnitude of the association was attenuated but remained significant after adjustment for conventional risk factors [12].

SEP has been measured with education, living conditions, occupation, or income respectively in many studies, or with a combination of socioeconomic determinants. These socioeconomic determinants are related to resources as well as exposure and susceptibility to diseases. Health behavior and psychological distress were considered two major pathways by which SEP may influence levels of inflammatory biomarkers. More recent studies have documented the potential links between SEP at multiple points in a life course and its association with the development of cardiovascular diseases [13, 14]. Childhood SEP has been defined frequently according to the parent's education and occupation, household income, or living conditions in early life. SEP in young adulthood has often been defined according to personal education attainment. Personal employment, income, wealth, or housing conditions were used to defined the SEP time point of active professional life or middle or older age [15]. Frameworks have been conceptualized to illustrate SEP throughout a life course [16-18]. The most frequently tested hypotheses of life-course SEP and health were the accumulation of risk, sensitive (or critical) periods, and social mobility. The accumulation risk model focuses on the total cumulative exposure to SEP throughout a life course, whereas the sensitive or critical period entails the SEP of a certain period in a life course having stronger effects than those of other periods. The social mobility model describes the trajectories of SEP that people are involved in through their life span and is often defined as inter-generational movements of social position from family of origin to adulthood. The pattern of social mobility were found to be significantly associated with health in later life. The health of people who remained stable in an advantaged position is likely to be better when compared to that of those who moved upward from a disadvantaged to an advantaged position. The health of who remained in the disadvantaged position is likely to be worse than that of those who moved upward from a disadvantaged to an advantaged social position [19]. A growing body of literature demonstrates the links between life-course SEP and inflammatory markers among Western populations; however, most studies have attempted to examine the critical period or accumulation hypothesis [20-23]. Controversial or negative findings were reported because of limited time points of SEP measures. For those that examined the social mobility hypothesis, the trajectory of SEP has often been limited to two time points [17, 24]. Although the aforementioned life course frameworks have often been examined as competing theories, the interdependent nature of these frameworks has been suggested and evidence has shown that the extent to which these frameworks are supported between life course SES and cardiovascular risk varies across race/ ethnicity [14]. However, few studies have examined the association between SEP and inflammatory markers in Asian populations. Understanding the association between life-course SEP and the level of inflammation may provide insights into the persistent social patterning of cardiovascular risk and prevention. Studies based on representative cohorts and a life-course design, in particular those based on nonWestern populations, are warranted to elucidate the links between the SEP that people experience throughout their life course and inflammation later in life. Therefore, the aim of this study was to examine how life-course SEP predicts inflammatory biomarkers in older age. Life-course SEP frameworks, including the "accumulation of risk," "social mobility," and "sensitive periods," were examined to gain a better understanding of the effects of SEP on inflammatory biomarkers.

\section{Methods}

\section{Study population and protocol}

Data were obtained from the Social Environment and Biomarkers of Aging Study (SEBAS), which was an extension of the population-based Taiwan Longitudinal Survey of Aging (TLSA) that began in 1989 [25]. A random subsample of respondents who completed the 1999 TLSA survey were invited to participate in the first wave of SEBAS in 2000. Our analysis focused on the second wave of the SEBAS conducted in 2006, which comprised the surviving participants from SEBAS 2000, aged $\geq 60$ years in 2006 , and a randomly selected subset of a younger refresh cohort of the TLSA in 2003, aged 50-57 in 2003 and 53-60 in 2006, resulting in a nationally representative sample of adults aged $\geq 53$ years. The nationally representative sampling design, age composition of the initial and refresh cohorts, and response rates of this prospective cohort have been described previously [26, 27]. The survey protocols of the SEBAS were approved by human subject committees at the Bureau of Health Promotion (Taipei, Taiwan; reformed into the Health Promotion Administration, Ministry of Health and Welfare, Taiwan, in 2013. Official Approval Code: NIFP-IRB2000-01), Georgetown University (Washington, D.C., USA. Official Approval Code: 1999-195), and 
Princeton University (Princeton, New Jersey, USA. Official Approval Codes: \#1848, \#2193, \#2791, \#3391). It consisted of an in-home face-to-face questionnaire interview, an in-hospital physical examination, and laboratory assays of biomarkers based on a fasting blood specimen. Informed consent was obtained before participation. Sociodemographic variables collected through face-to-face interviews at recruitment of the TLSA were analyzed with the SEBAS and survival data. Data on socio-demographic characteristics (age, sex, and educational attainment), lifestyle (smoking, alcohol drinking, dietary patterns, and exercise), disease at baseline, and family history of stroke and heart-related disease were collected via face-to-face interviews using a structured questionnaire. Anthropometric measurements of weight and height were performed in hospital. Body mass index (BMI) was calculated using body weight in kilograms divided by the square of height in meters. The classification of BMI conformed to the categories determined by the Taiwan Ministry of Health and Welfare. Systolic and diastolic blood pressures were calculated as the average of two seated readings. The two readings were taken $1 \mathrm{~min}$ apart by a registered nurse using a mercury sphygmomanometer on the right arm of the individual at least $20 \mathrm{~min}$ after the respondent arrived at the hospital. Levels of other biomarkers including total cholesterol, high-density lipoprotein (HDL) cholesterol, and triglycerides were determined at the central laboratory from the fasting blood samples. Low-density lipoprotein (LDL) was estimated indirectly from the concentrations of total cholesterol, HDL cholesterol, and triglycerides.

\section{SEP throughout a life course}

This study takes the life course approach by incorporating exposure in early childhood to adulthood and older age. Variables reflecting SEP at four time points throughout a life course were selected following the theatrical basis and stages proposed by Galobardes [15]. The SEP at the time points was measured according to the father and participant's education or occupation and a summarized indicator of socioeconomic status. The level of education and major life occupation were collected according to the participant's self-reported response to the face-to-face questionnaire interview from the baseline survey. The New Occupational Prestige and Socioeconomic Score for Taiwan [28] was applied to categorize the father's occupation and the participant's own occupation into groups that ranked from supervisor/executive/professional, technical/semiprofessional, and skilled worker/clerical/sales to unskilled worker and unemployed.

\section{Childhood SEP (father's education and occupation)}

Childhood SEP was defined as SEP at 6 years of age and measured according to the father's education and occupation. The variable was categorized into three groups: low $=0$ years, medium $=1-5$ years, high $=6$ years or more. Occupation was categorized as low $=$ unskilled worker or unemployed, medium $=$ technical $/$ semiprofessional or skilled worker/clerical/sales, and high = executive/ professional/supervisor. These education and occupation categorizations were combined into only two or three categories for analyses. The three categories of childhood SEP for descriptive analyses were low (father's education = low and father's occupation $=$ low), high (father' education $=$ high $/$ medium and father's education $=$ high) and medium (those who were not in low or high categories). These three categories were further grouped into dichotomous categories for trajectory analysis: relatively low (low or medium) and relatively high (high).

\section{Young adulthood SEP (own education attainment)}

Young adulthood SEP was defined as SEP at age 25, the age at which most Taiwanese people complete their education, and was measured according to personal education attainment. The variable was first categorized into low, medium, and high identically to how the father's education was categorized. The three categories of young adulthood SEP for descriptive analyses were low $=0$ years, medium $=1-5$ years, and high $=6$ years or more. The dichotomous categories for trajectory analysis were relatively low (low or medium) and relatively high (high).

\section{Active professional life SEP (own education attainment and occupation)}

Active professional life SEP was defined as SEP at age 40, and measured according to the participant's own education and major occupation in life. Age 40 was defined arbitrarily because it was the mid-point between 25 and 55 years because age 25 was the age at which most Taiwanese people complete their education, and age 55 is around the average age (55.9) at which Taiwanese people retired [29]. A participant's own occupation was first categorized into low, medium, and high identically to how the father's occupation was categorized. The three categories of active professional life SEP for descriptive analyses were low (own education $=$ low and own occupation $=$ low), medium (own education $=$ medium or own occupation = medium), and high (own education $=$ high and own education $=$ high). The three categories were further grouped into dichotomous categories: relatively low (low or medium) and relatively high (high). 


\section{Older age SEP (summarized indicator of self-related socioeconomic status)}

The final point was SEP at the time when the blood samples for inflammatory marker assays were collected. The participant ages ranged from 53 to 96 years. A summarized SEP indicator of self-rated socio-economic status was used to measure SEP at this time point. This instrument, known as the MacArthur Scale of Subjective Social Status, asks respondents to use 10 rungs of a ladder to position themselves socioeconomically relative to other people in their country. This subjective measure has been demonstrated to be a significant predictor of health even in the presence of conventional objective measures of social economic status for older Taiwanese [30]. The response to the question was categorized into low $=0-4$ rungs, medium $=5$ rungs, high $=6-10$ rungs and then further categorized into relatively high $=5-10$ rungs and relatively low $=1-4$ rungs.

\section{Inflammatory markers}

The inflammatory markers examined in this study were CRP and interleukin (IL-6). All participants were instructed to fast for at least $8 \mathrm{~h}$ before their hospital visit. Venous blood samples were drawn and centrifuged on site according to standard operating procedures. High-sensitivity CRP was determined by immunoturbidimetry assay (SIEMENS Healthcare Diagnostics, Tarrytown, NY, USA). The lower limit of detection was $0.012 \mathrm{mg} / \mathrm{dL}$ for CRP, and the interassay coefficient of variation $(\mathrm{CV})$ reported by the lab was $2.77 \%$. We measured IL-6 using enzyme-linked immunosorbent assay (R\&D Systems, Inc., Minneapolis, MN, USA) with a detection limit of $0.7 \mathrm{pg} / \mathrm{mL}$, and the interassay CV reported by the lab was $12.6 \%$. Aliquots of serum were kept frozen at $-70{ }^{\circ} \mathrm{C}$ until a batch assay of IL-6 was performed in 2007 and CRP in 2009 in the central lab. Duplicate assays were performed and the average of two readings was computed to reduce variation in the lab results, as reported elsewhere [31]. When the value was reported by the laboratory as below assay sensitivity (BAS) for one or both duplicate assays, we coded the BAS values to the detection limit before calculating the mean between duplicate assays. The outliers were trimmed by recoding them to p75 $+5 \times \mathrm{IQR}$, where p75 indicates the 75 th percentile, and IQR is the interquartile range (75th-25th percentile).

\section{Covariates}

Risk factors for cardiovascular disease, including age (53-74; 75-84; and $\geq 85$ ), gender, ever being diagnosed with hypertension (yes/no), diabetes (yes/ no), heart-related disease (yes/no), stroke (yes/no), Framingham risk score (cont.), lifestyle and health behavior, such as body mass index (BMI; underweight, normal, overweight, and obese), smoking (nonsmoker, current smoker, and ex-smoker), alcohol drinking (nondrinker, occasional drinker, and frequent drinker), five servings of vegetable and fruit (no/yes), exercise (none, 1-2 times/week, and >3 times/week), and depression symptoms were considered potential confounders of the multiple adjustment analysis. Depression symptoms were determined according to the respondent's score on the Center for Epidemiology Studies Depression Scale (CES-D). Perceived stress was measured with a 10 item Perceived Stress Scale, which was the 4-item shortened version of the Perceived Stress Scale to assess stress in the past 2 weeks, using a 1-5 response scale [32]. Because of missing data of some of the items, score was calculated by averaging across the available items. Higher values reflected greater perceived stress. The Framingham Risk Score already takes into account age, sex, smoking, hypertension, HDL, and cholesterol; consequently, these variables were not added individually to the multiple adjusted models.

\section{Statistical analysis}

A total of 1036 participants with available SEP data for at least of one time point were included in the current study. All estimates and standard errors were weighted to adjust for oversampling and unequal response rates by age, gender, and other covariates. Mean and standard deviation were presented for continuous variables with ANOVA tests for differences among the low, medium and high groups. Percentages were presented for categorical data and differences among the three SEP groups were tested by chi square analysis. In addition to the descriptive analysis of the participant by using SEP, we identified the distinct trajectories of life-course SEP; then used the trajectory group membership of each participant for subsequent analyses. Life-course SEP trajectory groups were determined using group-based trajectory modeling [33]. Analysis was implemented in PROC TRAJ of SAS software, which is a finite mixed modeling application that uses trajectory groups to statistically approximate unknown trajectories among population members [34]. The group-based trajectory approach we used involved fitting a polynomial model for each group. SEP of each group of participants over time is described using the binary logit distribution. The probability of staying in high SEP is directly measured through the proportion of individuals within each group staying in high SEP at a given time point. A total of 988 participants with available SEP data for four time points were classified into different trajectory groups according to the highest posterior probabilities of belonging to each group. The optimal number of groups was determined by comparing the Bayesian information criteria (BIC) and the principle of parsimony [35]. Multivariable 
linear regressions were then used to compare the levels of IL- 6 and CRP of individuals in different trajectory groups.

\section{Results}

Table 1 shows the characteristics of the SEBAS study population by childhood, young adulthood, active professional life, and older-age SEP. SEP at different time points of a life course were correlated. Percentages of low childhood SEP were higher among the low young adulthood SEP, low active professional SEP, and low older age SEP groups. Compared with participants of low or median SEP, those who were in the high SEP category had the lowest level of CRP and IL-6. Significant differences were observed among categories of young adulthood SEP, active professional life SEP, and older age SEP for CRP and between categories of young adulthood SEP and active professional life SEP for IL-6. There were no consistent and statistically discernible differences with regard to lifestyle factors such as smoking, alcohol consumption, having five servings of vegetable and fruit per day, or exercise across various SEP categories. The proportion of people having depressive symptoms were lower among the higher SEP categories.

Three distinct SEP trajectory groups were determined to describe the pattern of social mobility that the participants were involved in through their life span from family of origin to adulthood and older age (Fig. 1). Group 1 (Low-Low) represented 36.5\% of the participants who remained in a relatively lower SEP among the four time points; Group 2 (Low-High) comprised $26.8 \%$ of the participants who showed an upward mobility of life-course SEP from childhood to later in life; Group 3 consisted of the remaining $36.7 \%$ of participants who stayed in a relatively higher SEP throughout their life course. The results showed a social gradient in level of inflammation that runs from top to bottom of the SEP trajectories. A descending trend in levels of multiple adjusted CRP from $0.125 \mathrm{mg} / \mathrm{dL}$ [95\% confidence interval (CI): $0.112-0.140 \mathrm{mg} / \mathrm{dL}]$ and $0.121 \mathrm{mg} / \mathrm{dL}$ (95\% CI: $0.108-0.136 \mathrm{mg} / \mathrm{dL}$ ) to $0.095 \mathrm{mg} / \mathrm{dL}$ (95\% CI: 0.085-0.105 mg/dL) was observed from Low-Low and Low-High to High-High. The levels of IL-6 were $2.598 \mathrm{pg} / \mathrm{mL}$ (95\% CI: $2.410-2.801 \mathrm{pg} / \mathrm{mL}$ ), $2.644 \mathrm{pg} / \mathrm{mL}$ (95\% CI: $2.436-2.869 \mathrm{pg} / \mathrm{mL}$ ), and $2.284 \mathrm{pg} / \mathrm{mL}$ (95\% CI: 2.125-2.454 pg/mL) for the Low-Low, Low-High, and High-High groups, respectively. No obvious increasing or decreasing trends were observed (Fig. 2). The participants in the High-High group had the lowest levels of CRP and IL-6. Compared with those in the Low-Low group, the participants in the Low-High group had a similar adjusted CRP (-0.032 ln mg/L; 95\% CI: -0.193, 0.128); participants in the High-High group had a significantly lower level of adjusted CRP concentration $(-0.279 \mathrm{ln} \mathrm{mg} / \mathrm{L} ; 95 \% \mathrm{CI}$ :
$-0.434,-0.125)$. Likewise, the participants in the HighHigh group had a significantly lower level of adjusted IL-6 concentration $(-0.129 \mathrm{ln} \mathrm{pg} / \mathrm{mL} ; 95 \% \mathrm{CI}-0.236$, $-0.023)$. The coefficients attenuated but remained nearly the same after we controlled for gender, age, BMI, established risk factors, depressive symptoms, and stress. (Table 2).

\section{Discussion}

We found three distinct life-course SEP groups by using the time points of childhood, young adulthood, active professional life and older age. Rapid industrialization and economic growth in Taiwan was recorded during the latter half of the twentieth century [36]. Higher education has expanded rapidly since the 1960s [37]. Industrialization and economic growth would have resulted in improvement in education and occupation status of the whole population and might explain why trajectories across four time points fit into only three groups and why a "high-low" group was not classified.

Low SEP in childhood was associated with elevated CRP and IL-6 in older age. Similar to those who remained in lower SEP among the four time points, the respondents who transited from low SEP in childhood and young adulthood to high SEP in active professional life had higher CRP and IL-6 in older age. Although the relationships between SEP and IL-6 levels were nonsignificant after adjustment for CRP, directionality of the relationships was observed.

Previous observational studies have demonstrated that low SEP was associated with higher inflammatory biomarkers. In a cross-sectional study on 2729 middle-aged participants from the U.S. Framingham Offspring Study cohort, the number of years of education was inversely associated with CRP (beta for logarithmic CRP: -0.0034 , $p<0.001$ ) and IL-6 (beta for logarithmic IL-6: -0.011, $p<0.07)$ levels after adjustment for biological and psychosocial confounders [9]. The findings of the current study are similar, suggesting that only CRP had a significant association, but not IL-6. In another cross-sectional study on 8998 hospital-based, healthy, and elite executive participants, a significant inverse association between the number of school years and CRP was reported. After adjustment for multiple potential confounders, the percentages of mean change in CRP levels for each additional school year were $-2.3 \%$ (95 CI: $-3.2 \%$ to $-1.3 \%$ ) for men and $-2.0 \%$ (95 CI: $-3.1 \%$ to $-0.8 \%$ ) for women [38]. Our findings are compatible with the aforementioned studies. Participants who remained in a high SEP throughout their life course had the lowest levels of CRP and IL- 6 concentrations.

Several studies have reported associations between adverse childhood experiences and chronic or age-related conditions. However, no consensus is reached in the 


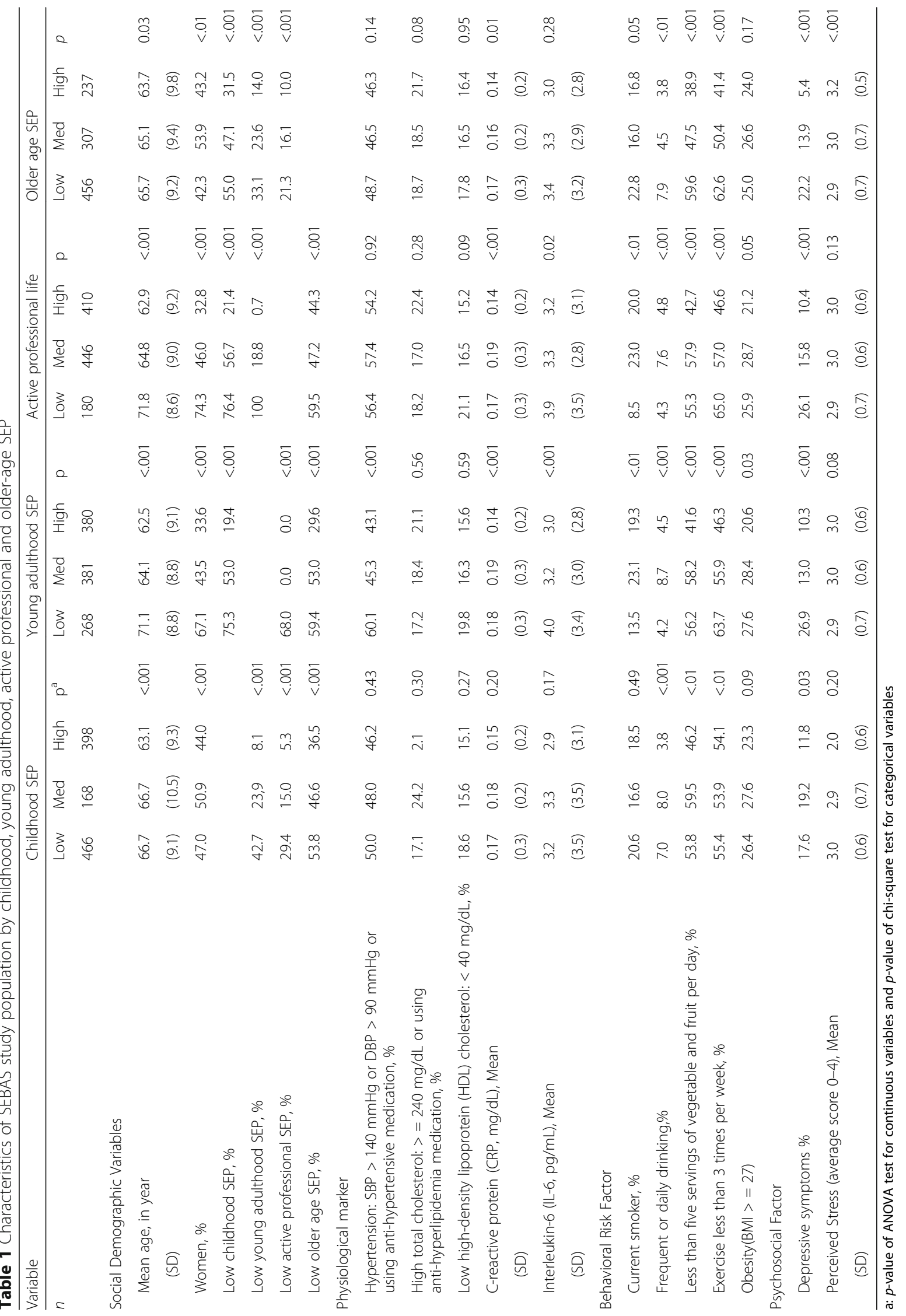




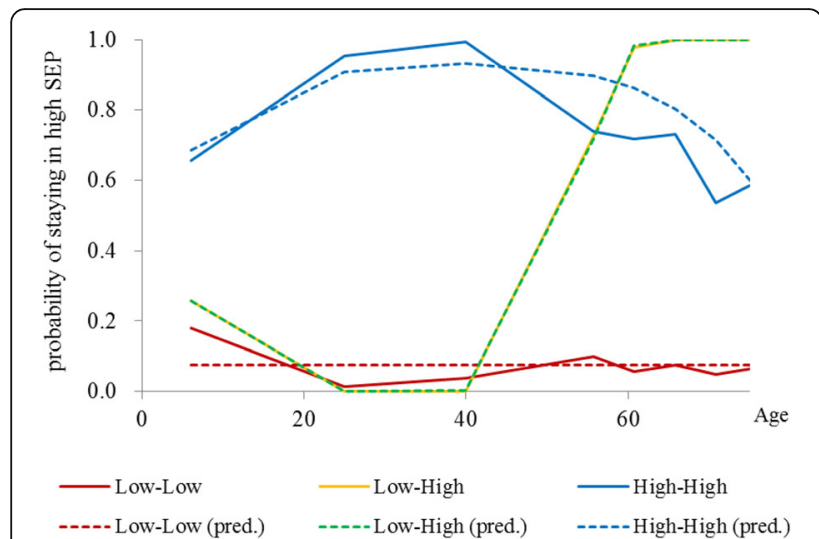

Fig. 1 Group-based trajectories of life-course SEP. BIC $=-2094.78$ (3900 observations); BIC $=-2088.60$ (988 persons). Group 1, LowLow (36.5\%); Group 2, Low-High (26.8\%); Group 3, High-High (36.7\%)

literature regarding whether childhood SEP or recent SEP is more strongly related to inflammatory biomarkers. In a cohort study comprising 12,681 Caucasian and African American people, cumulative exposure to lower SEP throughout a life course and low adult SEP

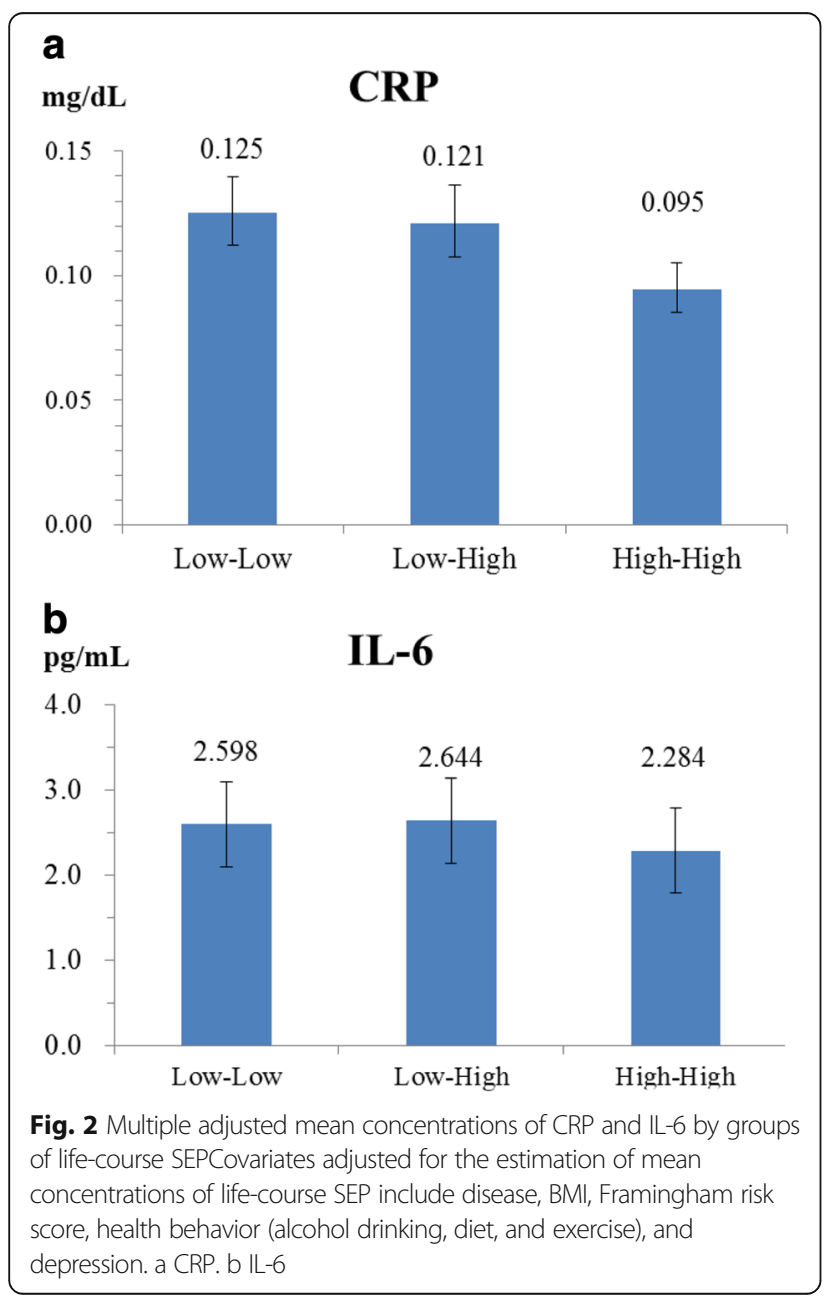

were associated with elevated CRP levels [39]. Furthermore, among the 5951 respondents of the British birth cohort study, significantly inverse associations were observed for childhood and early adulthood but not for SEP in older age [40]. A follow up study of the British birth cohort suggested that childhood socioeconomic position acts either as a sensitive period or as part of a cumulative life course model [13]. Our data support that early childhood SEP was a critical period and had stronger effects than those of other periods. The upward social mobility in adulthood could be attributed to the occupational prestige gained from active professional life and may have only marginal effects on inflammation in older age.

Health behavior and psychological distress were reported to be the two major pathways by which SEP may influence the levels of inflammatory biomarkers [41, 42]. An adverse childhood experience predicts an elevated risk of depression, a clustering of metabolic risk factors, and elevated inflammation levels in adult life. In a crosssectional study on 2266 randomly selected samples in Greece, those who reported "high" education had 45\% lower CRP, but the association was mainly explained by the adoption of an unhealthy lifestyle, such as increased smoking habits, physical inactivity, and obesity [43]. In a study on 851 men and women aged 30-54 years, SEP was no longer associated with IL-6 after adjustment for lifestyle factors [8]. The results of the current study show no consistent and statistically discernible differences with regard to lifestyle factors such as smoking, alcohol consumption, having five servings of vegetable and fruit per day, or exercise across various SEP categories. Higher SEP was associated with lower percentages of depressive symptoms. The associations between lifecourse SEP and CRP or IL-6 were attenuated but remained significant after we controlled for gender, age, BMI, established risk factors, depressive symptoms, and stress. The results are consistent with the findings of the 1958 British birth cohort study, showing that controlling for BMI, smoking, and physical activity had little effect on the associations between SEP and CRP [40].

CRP is a marker of systemic inflammation, and IL-6 acts as both a pro-inflammatory and anti-inflammatory cytokine in mediating inflammation. Levels of IL-6 and CRP are physiologically linked because of the function of IL- 6 on hepatic synthesis and the excretion of CRP. Markers of systemic inflammation, pro-inflammatory cytokines and anti-inflammatory cytokines may reflect different features of atherosclerotic processes and the interplay between life-course SEP and inflammation; thus, both CRP and IL-6 were associated with lifecourse SEP after adjustment for the covariates. The biological mechanisms through which SEP exerts its effects on inflammation is limited. Low SEP across the life- 


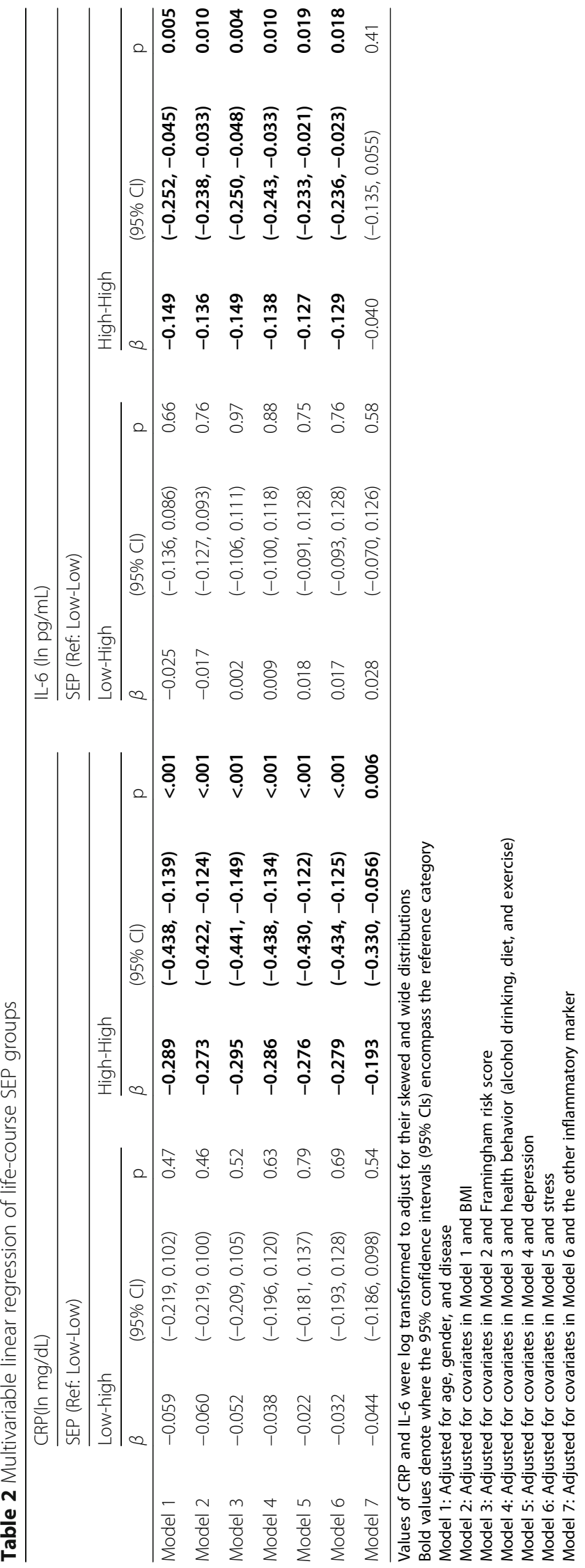


course was reported to be associated with increased inflammatory activity, and may have an impact on health via inflammation through the interconnection of lifestyle factors and genetic regulation of immune function. A study on Italian participants of a large scale prospective cohort in Europe demonstrated that indicators of social economic status were associated with DNA methylation of genes involved in inflammation and suggested that social adversity represented by low economic status might leave an epigenetic mark on cells, which would exacerbate the inflammatory response [24].

The strengths of this study are as follows: First, we used semi-longitudinal data based on subjective recall from a nationally representative cohort to provide an enhanced opportunity to examine how life-course SEP predicts inflammation in older age. Second, we use repeated measurement of SEP at four time points throughout a life course. Third, in contrast to other studies observing the overall pattern among the available time points, we used group-based trajectory modeling to identify distinct social mobility groups of SEP throughout a life course. This novel statistical approach provides clearer insight into hypothesis testing within the proposed frameworks. In addition to the subjective measure of SEP with education and occupation, we used an objective measure of SEP that has been demonstrated to be a significant predictor of health, even in the presence of conventional measures of SEP for older Taiwanese people [30]. Nevertheless, the limitations of this study must be considered in interpreting the findings. First, the inflammatory markers and covariates were measured at a single time point and may not accurately capture the time-varying nature of the variables. Second, only the father's education and occupation were collected for the cohort study. Admittedly, we were not able to consider the mother's education and occupation for the analysis. However, during the participants' childhood, not many women in Taiwan's patriarchal society were provided with education, and few of them had jobs. Therefore, the fathers' education and occupation were more practical choices for measuring the participants' SEP in childhood. In addition, data collection on infectious illnesses and conditions associated with elevated inflammatory markers was limited in this study and may have resulted in residual confounding due to the systemic nature of CRP and IL-6. No hard endpoints, such as cardiovascular morbidity or deaths, were analyzed.

The main focus of this study was to identify the possible upward, downward or stable trajectories for later modelling. The identification of trajectory groups is an inductive approach that allows the data to 'speak for themselves' by identifying groups of individuals whose SEP follows a similar course over time, highlighting differences and similarities between these individuals which call for explanation, and providing a foundation on which later modelling can be based. However, due to the constraints of limited sample size, we could just dichotomize the four SEP variables at different life stages.

\section{Conclusions}

In conclusion, life-course SEP is related to inflammation in older age. Low SEP in childhood is associated with elevated inflammatory markers in older age. Even after transit from low SEP in childhood to high SEP in older age, the risk remains. This study demonstrates the social inequalities in population health. The health of people who remained stable in an advantaged SEP is better when compared to that of those who remained in the disadvantaged SEP or those who upwardly moved from a disadvantaged position to an advantaged position. Our data support the notion that childhood SEP may act either as a sensitive period or as part of the accumulation of risk. Intervention in early childhood and strategies for ensuring that every child has an optimal start in life are crucial for reducing the burdens of inflammation.

\begin{abstract}
Abbreviations
BAS: Below assay sensitivity; BMI: Body mass index; CES-D: Center for Epidemiology Studies Depression Scale (CES-D); Cl: Confidence interval; CRP: C-reactive protein; CV: Coefficient of variation; DBP: Diastolic blood pressure; HDL: High-density lipoprotein cholesterol; IL-6: Interleukin-6; IQR: Interquartile range; p75: The 75th percentile; SBP: Systolic blood pressure; SD: Standard deviation; SEBAS: Social Environment and Biomarkers of Aging Study; SEP: Socioeconomic position; TLSA: Taiwan Longitudinal Survey of Aging
\end{abstract}

\section{Acknowledgements}

We thank the participants and fieldwork staff for their contributions to the study.

\section{Funding}

The Social Environment and Biomarkers of Aging Study (SEBAS) is supported by the US National Institute on Aging, National Institutes of Health (grants R01AG16790 and R01AG16661), and the Taiwan Bureau of Health Promotion, Department of Health (reformed into the Health Promotion Administration, Ministry of Health and Welfare, Taiwan, in 2013). Data and documentation for the 2000 and 2007 Social Environment and Biomarkers of Aging Study are available from the Inter-University Consortium for Political and Social Research at https://doi.org/10.3886/ICPSR03792.v7.

\section{Availability of data and materials}

The datasets generated and/or analysed during the current study are not publicly available due to the terms of consent to which the participants agreed but data are however available from the authors upon reasonable request and with permission of the Health Promotion Administration at the Ministry of Health and Welfare in Taiwan.

\section{Authors' contributions}

$\mathrm{YL}, \mathrm{MJ}$ and $\mathrm{KC}$ designed the study, developed the methodology. $\mathrm{YL}$ performed the analysis, wrote the manuscript. All authors approved the final version of the manuscript for submission. 


\section{Ethics approval and consent to participate}

The study protocols were approved by Institutional Review Boards at the Bureau of Health Promotion (Taichung, Taiwan. Official Approval Code: NIFP-IRB-2000-01), at Georgetown University (Washington, DC. Official Approval Code: 1999-195), and Princeton University (Princeton, New Jersey. Official Approval Codes: \#1848, \#2193, \#2791, \#3391). Written informed consent was obtained before participation from participants or their legal proxies.

\section{Consent for publication}

Not applicable.

\section{Competing interests}

The authors declare that they have no competing interests.

\section{Publisher's Note}

Springer Nature remains neutral with regard to jurisdictional claims in published maps and institutional affiliations.

\section{Author details}

${ }^{1}$ Health Promotion Administration, Ministry of Health and Welfare, Taipei, Taiwan. ${ }^{2}$ Eli Lilly and the company, Surrey, UK. ${ }^{3}$ Institute of Epidemiology and Preventive Medicine, College of Public Health, National Taiwan University, Mailing address: Rm. 517, 5F., No. 17, Xuzhou Rd., Zhongzheng Dist, Taipei City 100, Taiwan.

Received: 19 January 2016 Accepted: 24 August 2017

Published online: 02 September 2017

\section{References}

1. Kaplan GA, Keil JE. Socioeconomic factors and cardiovascular disease: a review of the literature. Circulation. 1993;88(4 Pt 1):1973-98.

2. Marmot MG, Rose G, Shipley M, Hamilton PJ. Employment grade and coronary heart disease in British civil servants. J Epidemiol Community Health. 1978;32(4):244-9.

3. Pearson TA, Mensah GA, Hong Y, Smith SC Jr. CDC/AHA workshop on markers of inflammation and cardiovascular disease: application to clinical and public health practice: overview. Circulation. 2004;110(25):e543-4.

4. Iso H, Cui R, Date C, Kikuchi S, Tamakoshi A. C-reactive protein levels and risk of mortality from cardiovascular disease in Japanese: the JACC study. Atherosclerosis. 2009;207(1):291-7.

5. Hamer M, Chida Y, Stamatakis E. Association of very highly elevated Creactive protein concentration with cardiovascular events and all-cause mortality. Clin Chem. 2010;56(1):132-5.

6. Kaptoge S, Di Angelantonio E, Lowe G, Pepys MB, Thompson SG, Collins R, Danesh J. C-reactive protein concentration and risk of coronary heart disease, stroke, and mortality: an individual participant meta-analysis. Lancet. 2010;375(9709):132-40.

7. Abeywardena MY, Leifert WR, Warnes KE, Varghese JN, Head RJ. Cardiovascular biology of interleukin-6. Curr Pharm Des. 2009;15(15):1809-21.

8. Petersen KL, Marsland AL, Flory J, Votruba-Drzal E, Muldoon MF, Manuck SB. Community socioeconomic status is associated with circulating interleukin-6 and C-reactive protein. Psychosom Med. 2008;70(6):646-52.

9. Loucks EB, Sullivan LM, Hayes LJ, D'Agostino RB Sr, Larson MG, Vasan RS, Benjamin EJ, Berkman LF. Association of educational level with inflammatory markers in the Framingham offspring study. Am J Epidemiol. 2006;163(7):622-8

10. Gruenewald TL, Cohen S, Matthews KA, Tracy R, Seeman TE. Association of socioeconomic status with inflammation markers in black and white men and women in the coronary artery risk development in young adults (CARDIA) study. Soc Sci Med. 2009;69(3):451-9.

11. Fraga S, Marques-Vidal P, Vollenweider P, Waeber G, Guessous I, Paccaud F, Barros $\mathrm{H}$, Stringhini S. Association of socioeconomic status with inflammatory markers: a two cohort comparison. Prev Med. 2014;71C:12-9.

12. Nazmi A, Victora CG. Socioeconomic and racial/ethnic differentials of Creactive protein levels: a systematic review of population-based studies. BMC Public Health. 2007;7:212.

13. Jones R, Hardy R, Sattar N, Deanfield JE, Hughes A, Kuh D, Murray ET, Whincup PH, Thomas C, Scientific N, et al. Novel coronary heart disease risk factors at 60-64 years and life course socioeconomic position: the 1946 British birth cohort. Atherosclerosis. 2015;238(1):70-6.
14. Walsemann KM, Goosby BJ, Farr D. Life course SES and cardiovascular risk: heterogeneity across race/ethnicity and gender. Soc Sci Med. 2016;152:147-55.

15. Galobardes B, Shaw M, Lawlor DA, Lynch JW, Davey Smith G. Indicators of socioeconomic position (part 1). J Epidemiol Community Health. 2006;60(1):7-12.

16. Pollitt RA, Rose KM, Kaufman JS. Evaluating the evidence for models of life course socioeconomic factors and cardiovascular outcomes: a systematic review. BMC Public Health. 2005;5:7.

17. Loucks EB, Pilote L, Lynch JW, Richard H, Almeida ND, Benjamin EJ, Murabito JM. Life course socioeconomic position is associated with inflammatory markers: the Framingham offspring study. Soc Sci Med. 2010;71(1):187-95.

18. Hallqvist J, Lynch J, Bartley M, Lang T, Blane D. Can we disentangle life course processes of accumulation, critical period and social mobility? An analysis of disadvantaged socio-economic positions and myocardial infarction in the Stockholm heart epidemiology program. Soc Sci Med. 2004;58(8):1555-62.

19. Cable N. Life course approach in social epidemiology: an overview, application and future implications. J Epidemiol. 2014;24(5):347-52.

20. Pollitt RA, Kaufman JS, Rose KM, Diez-Roux AV, Zeng D, Heiss G. Early-life and adult socioeconomic status and inflammatory risk markers in adulthood. Eur J Epidemiol. 2007;22(1):55-66.

21. Packard CJ, Bezlyak V, McLean JS, Batty GD, Ford I, Burns H, Cavanagh J, Deans KA, Henderson M, McGinty A, et al. Early life socioeconomic adversity is associated in adult life with chronic inflammation, carotid atherosclerosis, poorer lung function and decreased cognitive performance: a crosssectional, population-based study. BMC Public Health. 2011;11:42.

22. Gruenewald TL, Karlamangla AS, Hu P, Stein-Merkin S, Crandall C, Koretz B, Seeman TE. History of socioeconomic disadvantage and allostatic load in later life. Soc Sci Med. 2012;74(1):75-83.

23. Danese A, McEwen BS. Adverse childhood experiences, allostasis, allostatic load, and age-related disease. Physiol Behav. 2012;106(1):29-39.

24. Stringhini S, Polidoro S, Sacerdote C, Kelly RS, van Veldhoven K, Agnoli C, Grioni S, Tumino R, Giurdanella MC, Panico S, et al. Life-course socioeconomic status and DNA methylation of genes regulating inflammation. Int J Epidemiol. 2015:44(4):1320-30.

25. Hermalin Al: The well-being of the elderly in Asia: a four-country comparative study: American mathematical soc.; 2002.

26. Cornman JC, Glei DA, Goldman N, Chang MC, Lin HS, Chuang YL, Hurng BS, Lin YH, Lin SH, Liu IW, et al. Cohort profile: the social environment and biomarkers of aging study (SEBAS) in Taiwan. Int J Epidemiol. 2014;45(1):54-63.

27. Goldman N, Glei DA, Lin YH, Weinstein M. Improving mortality prediction using biosocial surveys. Am J Epidemiol. 2009;169(6):769-79.

28. Hwang Y-J. The construction and assessment of the 'new occupational prestige and socioeconomic scores for Taiwan': the indigenization of the social science and sociology of education research. Bulletin of Educational Research. 2003;49(4):1-31.

29. Lu L. Attitudes toward older people and coworkers' intention to work with older employees: a Taiwanese study. Int J Aging Hum Dev. 2010;71(4):305-22.

30. Goldman N, Cornman JC, Chang MC. Measuring subjective social status: a case study of older Taiwanese. Journal of cross-cultural gerontology. 2006;21(1-2):71-89.

31. Glei DA, Goldman N, Lin YH, Weinstein M. Age-related changes in biomarkers: longitudinal data from a population-based sample. Research on aging. 2011;33(3):312-26.

32. Cohen S, Kamarck T, Mermelstein R. A global measure of perceived stress. J Health Soc Behav. 1983;24(4):385-96.

33. Nagin DS, Tremblay RE. Analyzing developmental trajectories of distinct but related behaviors: a group-based method. Psychol Methods. 2001;6(1):18-34.

34. Jones BL, Nagin DS. Advances in group-based trajectory modeling and an SAS procedure for estimating them. Sociol Methods Res. 2007;35(4):542-71.

35. Nagin D: Group-based modeling of development: Harvard University press; 2005.

36. Liu C, Armer JM. Education's effect on economic growth in Taiwan. Comp Educ Rev. 1993;37(3):304-21.

37. Wang RJ. From elitism to mass higher education in Taiwan: the problems faced. High Educ. 2003;46(3):261-87.

38. Steinvil A, Shirom A, Melamed S, Toker S, Justo D, Saar N, Shapira I, Berliner S, Rogowski O. Relation of educational level to inflammation-sensitive biomarker level. Am J Cardiol. 2008;102(8):1034-9. 
39. Pollitt RA, Kaufman JS, Rose KM, Diez-Roux AV, Zeng D, Heiss G. Cumulative life course and adult socioeconomic status and markers of inflammation in adulthood. J Epidemiol Community Health. 2008;62(6):484-91.

40. Tabassum F, Kumari M, Rumley A, Lowe G, Power C, Strachan DP. Effects of socioeconomic position on inflammatory and hemostatic markers: a life-course analysis in the 1958 British birth cohort. Am J Epidemiol. 2008;167(11):1332-41

41. McDade TW, Hawkley LC, Cacioppo JT. Psychosocial and behavioral predictors of inflammation in middle-aged and older adults: the Chicago health, aging, and social relations study. Psychosom Med. 2006;68(3):376-81.

42. Glymour MM, Clark CR, Patton KK. Socioeconomic determinants of cardiovascular disease: recent findings and future directions. Current Epidemiology Reports. 2014;1(2):89-97.

43. Panagiotakos DB, Pitsavos CE, Chrysohoou CA, Skoumas J, Toutouza M, Belegrinos D, Toutouzas PK, Stefanadis C. The association between educational status and risk factors related to cardiovascular disease in healthy individuals: the ATTICA study. Ann Epidemiol. 2004;14(3):188-94.

Submit your next manuscript to BioMed Central and we will help you at every step:

- We accept pre-submission inquiries

- Our selector tool helps you to find the most relevant journal

- We provide round the clock customer support

- Convenient online submission

- Thorough peer review

- Inclusion in PubMed and all major indexing services

- Maximum visibility for your research

Submit your manuscript at www.biomedcentral.com/submit 